\section{Three-dimensional apoptotic nuclear behavior analyzed by means of Field Emission in Lens Scanning Electron Microscope}

\author{
S. Salucci, S. Burattini, E. Falcieri, \\ P. Gobbi \\ Department of Earth, Life and \\ Environmental Sciences, University of \\ Urbino Carlo Bo, Italy
}

\begin{abstract}
Apoptosis is an essential biological function required during embryogenesis, tissue homeostasis, organ development and immune system regulation. It is an active cell death pathway involved in a variety of pathological conditions. During this process cytoskeletal proteins appear damaged and undergo an enzymatic disassembling, leading to formation of apoptotic features. This study was designed to examine the threedimensional chromatin behavior and cytoskeleton involvement, in particular actin re-modeling. HL-60 cells, exposed to hyperthermia, a known apoptotic trigger, were examined by means of a Field Emission in Lens Scanning Electron Microscope (FEISEM). Ultrastructural observations revealed in treated cells the presence of apoptotic patterns after hyperthermia trigger. In particular, three-dimensional apoptotic chromatin rearrangements appeared involving the translocation of filamentous actin from cytoplasm to the nucleus. FEISEM immunogold techniques showed actin labeling and its precise three-dimensional localization in the diffuse chromatin, well separated from the condensed one. The actin presence in dispersed chromatin inside the apoptotic nucleus can be considered an important feature, indispensable to permit the apoptotic machinery evolution.
\end{abstract}

\section{Introduction}

Apoptosis is tightly genetically regulated, and plays a pivotal role for host defenses and cancer suppression. ${ }^{1}$ Inversely, apoptosis down-regulation can promote carcinogenesis and tumor progression. Many anticancer therapies work by inducing apoptosis in cancer cells. ${ }^{2}$ Hyperthermia is usually applied as an adjunct to an already established treatment modality (especially radiotherapy and chemotherapy), where tumor temperatures in the range of $40-43^{\circ} \mathrm{C}$ are reached. In several phase-III trial clinical, an improvement of both local control and survival rates have been demonstrated by adding hyperthermia to radiotherapy in patients with locally advanced or recurrent superficial and pelvic tumors. ${ }^{3}$

Hyperthermia, a known apoptotic inducer in various cell lines in vitro ${ }^{4-7}$ usually determines typical apoptotic features characterized by nuclear chromatin reorganization with the cytoskeleton involvement. It has been shown that cytoskeleton reorganization takes place during cell proliferation, maturation and differentiation. ${ }^{8,9}$ Actin filaments are a major component of the cytoskeleton and play an important role in cell migration, growth, cytokinesis, endocytosis, cell shape determination and vesicle trafficking. In cells, actin is found both in a monomeric form (G-actin) and in the form of filaments (F-actin), which can be arranged in bundles or networks. ${ }^{10-15}$ In recent years, it has been demonstrated that actin and actin-binding proteins play an important role in a variety of nuclear activities. ${ }^{16-18}$ Actin was discovered as a component of chromatin remodeling complex ${ }^{19,20}$ and transcription machineries, where it is incorporated into newly synthesized ribonucleoproteins. ${ }^{21-23}$ Moreover, it influences a long-range chromatin organization ${ }^{24}$ even if mechanisms of actin participation in many nuclear processes are highly distinct from those correlated to chromatin modification, and may involve actin polymerization. ${ }^{20}$ In this context, previous reports showed not only the presence of $\mathrm{F}$ actin in the cell nuclei of various cell lines treated with different cytostatic drugs, but also its contribution to chemotherapeutic and radiotherapic-induced cell death. ${ }^{25-27}$ It is known that apoptotic nuclear changes occur in the presence of deep reorganization of nuclear matrix. In particular, the nuclear lamina undergoes a progressive depolarization which lead to a spatial redistributions of its components. ${ }^{5}$ Nevertheless, the function and localization of actin in the nucleus is not yet fully characterized.

Here, we would like to provide further evidence of hyperthermia-induced nuclear and cytoplasmic F-actin reorganization in HL-60 cells by means of a Field Emission in Lens Scanning Electron Microscope (FEISEM), able to show, in an accurate way, the three-dimensional nuclear filament bundles and their reorganization and management during the apoptotic process.

\section{Materials and Methods}

\section{Cell culture and apoptosis induction}

HL60 human promyelocytic cells have been conventionally grown. Briefly, they have been cultured in RPMI 1640 supplemented with 10\%
Correspondence: Dr. Sara Salucci, Department of Earth, Life and Environmental Sciences, University of Urbino Carlo Bo, Via Ca' le Suore 2, 61029 Urbino (PU), Italy.

Tel. +39.0722.304248 - Fax: +39.0722.304244.

E-mail: sara.salucci@uniurb.it

Keywords: Chromatin; F-actin; immunogold; TEM; FEISEM.

Acknowledgments: particular thanks are due to Prof. M. Falconi and Dr. G. Teti (DIBINEM Bologna University) for the FEISEM facilities and their expert assistance.

Received for publication: 3 June 2015. Accepted for publication: 1 August 2015.

This work is licensed under a Creative Commons Attribution NonCommercial 3.0 License (CC BYNC 3.0).

\section{(C) Copyright S. Salucci et al., 2015}

Licensee PAGEPress, Italy

European Journal of Histochemistry 2015; 59:2539 doi:10.4081/ejh.2015.2539

heat-inactivated fetal calf serum, $2 \mathrm{mM}$ glutamine, $20 \mathrm{mM}$ Hepes, pH 7.5, and antibiotics (100 U/mL penicillin, $100 \mu \mathrm{g} / \mathrm{mL}$ streptomycin). They have been maintained in $5 \% \mathrm{CO}_{2}$ atmosphere at $37^{\circ} \mathrm{C}$ and cell viability has been assessed by the trypan blue exclusion test. Hyperthermia has been carried out for $1 \mathrm{~h}$ at $43^{\circ} \mathrm{C}$ in water bath followed by a recovery for $6 \mathrm{~h}$ at $37^{\circ} \mathrm{C} .{ }^{28}$

\section{Transmission electron microscopy analysis}

For conventional electron microscopy control and treated cells have been fixed with 2.5\% glutaraldehyde in phosphate buffer, postfixed in $1 \%$ Osmium Tetroxide $\left(\mathrm{OsO}_{4}\right)$ in the same buffer, alcohol dehydrated and embedded in araldite. Thin sections were stained by uranyl acetate and lead citrate. ${ }^{29}$ For immunogold actin investigation, treated and control specimens were fixed with $1 \%$ glutaraldehyde in $0.1 \mathrm{M}$ phosphate buffer for $1 \mathrm{~h}$, partially dehydrated until 70\% alcohol and embedded in London Resin White (LRWhite) (TAAB Laboratories Equipment Ltd., West Berkshire, UK) at $0^{\circ} \mathrm{C}$. Thin sections have been collected on 400 mesh nickel grids. After washing in distilled water and Tris buffer saline (TBS) and blocking not specific protein binding with normal goat serum (NGS) in $0.1 \%$ bovine serum albumin (BSA)/Tris buffer for $30 \mathrm{~min}$, the immunoreaction was carried out by means of a mouse antiactin monoclonal antibody (Boehringer, Mannheim, Germany) (1:100 in 0.1\% BSA/Tris buffer). The incubation was performed overnight at $4 \mathrm{C}$ in moist chamber. After washing in Tris buffer, the grids were incubated 
with a $10 \mathrm{~nm}$ colloidal gold conjugated antibody (1:100; BioCell) for $60 \mathrm{~min}$ at room temperature. After a further rinsing, the samples have been counterstained with uranyl acetate and lead citrate. All specimens have been examined with a Philips CM 10 electron microscope, at $80 \mathrm{KV} .{ }^{28,29}$

\section{FEISEM analysis}

Cell pellets were fixed with $1 \%$ glutaraldehyde in $0.1 \mathrm{M}$ phosphate buffer, $\mathrm{pH} 7.2$, cryopreserved with $2.3 \mathrm{M}$ sucrose in $0.1 \mathrm{M}$ phosphate buffer, $\mathrm{pH} 7.2$, frozen in liquid nitrogen, and cryosectioned in a Reichert JUNG FC 4/E (Leica, Vienna, Austria) cryoultramicrotome. Sections of 100-120 nm were mounted on silicon chips which were utilized as a specimen support. Samples were incubated overnight with the anti-F-actin antibody and then reacted with a secondary antibody (anti-mouse $\operatorname{IgG}$ ) conjugated with $10 \mathrm{~nm}$ colloidal gold particles (BioCell). Anti-F-actin-treated cryosections were then processed for FEISEM observation. They were post-fixed in $1 \%$ osmium tetroxide in phosphate buffer $0.15 \mathrm{M}$, pH 7.2, for $30 \mathrm{~min}$, dehydrated in an increasing ethanol series, and critical point-dried (CPD 030; Bal-Tec, Lichtenstein). The analysis was performed on uncoated samples with an FEISEM Jeol JSM 890 (Jeol; Tokyo, Japan) at $7 \mathrm{kV}$ accelerating voltage and $1 \times 10^{-11} \mathrm{~A}$ probe current, utilizing the secondary electrons image (SE), backscattered electrons image (BE) and a SE-BE mix. ${ }^{30}$

To check the amount of gold labeling and to avoid low marker efficiency due to steric hindrance of the gold particles, controls were performed by omitting incubation with the primary antibody.

\section{Results}

Both techniques, as expectedly, showed an high number of apoptotic cells, absent in control condition, confirming again that hyperthermia is a powerful apoptotic trigger. Both transmission electron microscopy (TEM) and FEISEM observations well described chromatin behavior in control cells and its changes during apoptosis in cells exposed to hyperthermia. In particular, FEISEM permitted to evidence the three-dimensional chromatin and cytoskeleton rearrangement which occurred under apoptotic condition. Analyses of cells at low magnification evidenced a preserved cellular morphology, with nuclear and cytoplasm components clearly detectable (Figure 1 A,B). At FEISEM, the nuclear matrix showed a typical tripartite structure with the nuclear lamina, the nuclear remnant and the inner fibrillary network well evident (Figure $1 \mathrm{C}, \mathrm{D})$. Moreover, it was possible clearly distinguish the condensed chromatin from the diffuse one (Figure 1E ) which appeared organized in a fibrillary network (Figure 1E) constituted mainly by $10 \mathrm{~nm}$ fibers surrounding areas of 80-100 nm (Figure 1F).

Cells exposed to hyperthermia showed the typical morphological apoptotic features: cytoplasm shrinkage and vacuolization as well as chromatin margination and condensation, usually in cup-shaped masses, appeared (Figure $2 \mathrm{~A}, \mathrm{~B}$ ). The latter are organized in dense patches scattered throughout nucleoplasm and around nucleus and separated from the diffuse chromatin (Figure 2C) which showed a behavior similar to that observed in control condition (Figure 2D). Inside the diffuse component, condensed chromatin clusters appeared similar to that observed in perinuclear area (Figure $2 \mathrm{E}, \mathrm{F}$ ). Luchetti et al. $^{5}$ demonstrated at TEM the presence of thin filaments in the diffuse chromatin of early apoptotic cells, absent in the control ones (Figure $3 \mathrm{~B}$, inset). They appeared organized in one or more bundles and for their aspect and size were similar to actin filaments (Figure $3 \mathrm{~A}$ ), then further characterized by TEM immunogold (Figure 3B). No data are available in literature on actin localization, by means of FEISEM, in the study of the three-dimensional apoptotic chromatin behavior. Immonogold technique revealed that untreated HL-60 cells showed a regularly organized chromatin and actin localized in cytoplasm, as demonstrated by gold particle deposition (Figure $3 \mathrm{C}$-E) .

In apoptotic cells, as described above, diffuse and condensed chromatin coexisted, but no labeling has been detected in the dense one. On the other hand, in diffuse chromatin
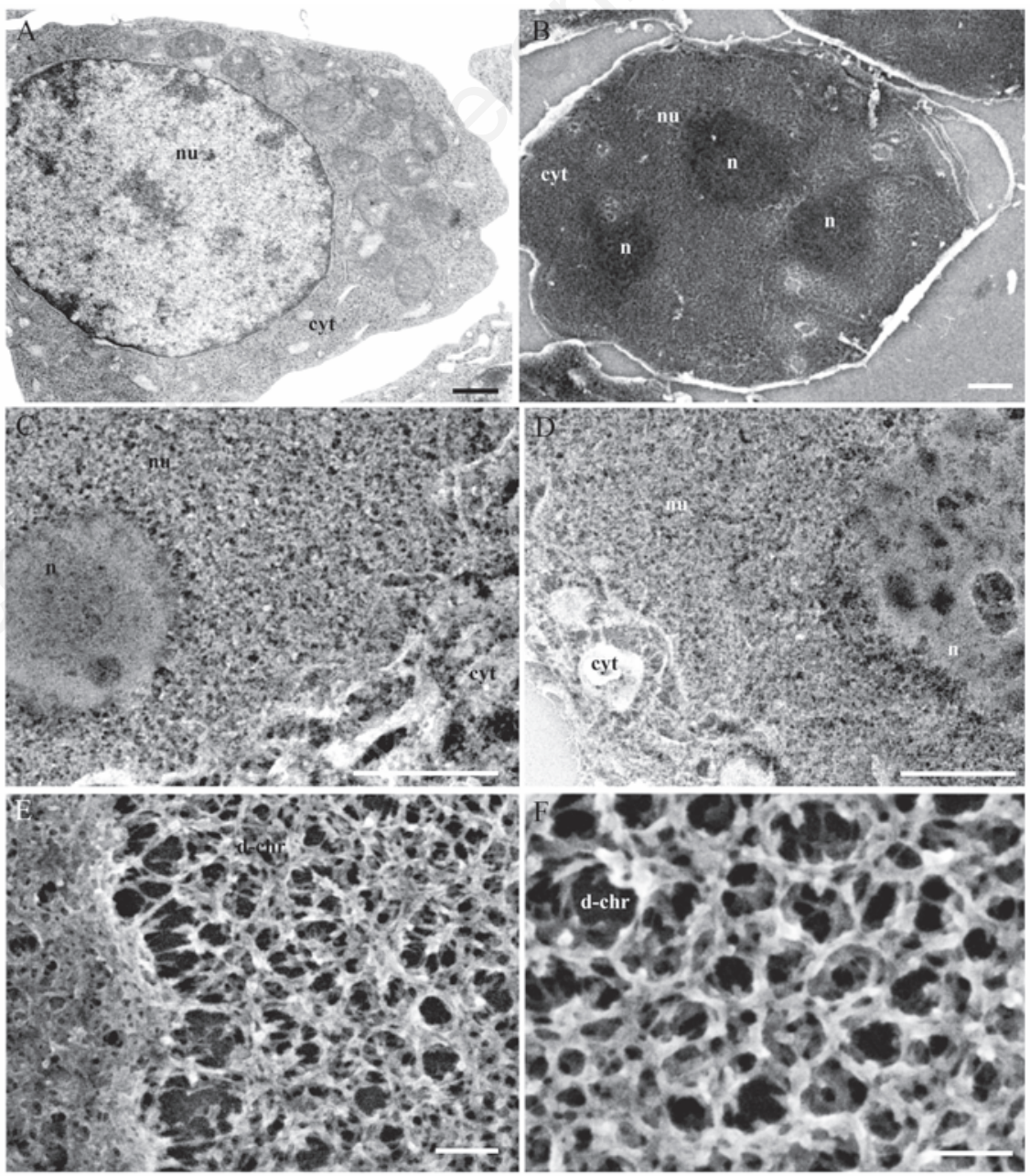

Figure 1. Control HL-60 cells at TEM (A) and FEISEM in SE image (B-F) analyses. Cytoplasm and nuclear structures appear well preserved (A, B). In particular, at FEISEM, nucleolus with its fibrillary components $(C, D)$, nuclear lamina and diffuse chromatin network are well detectable $(\mathrm{D}, \mathrm{E})$. This latter appears organized in $10 \mathrm{~nm}$ size fibers surrounding space of about 80-100 $\mathrm{nm}(\mathrm{F})$. $\mathrm{n}$, nucleolus; nu; nucleus; d-chr, diffuse chromatin; cty, cytoplasm. Scale bars: A-D) $1 \mu \mathrm{m}$; E) $100 \mathrm{~nm}$. 
gold particles appeared, organized in straight or curvilinear clusters, suggesting the presence of structures longer than $100 \mathrm{~nm}$ (Figure 3 F,G). Thus, between diffuse and condensed chromatin areas, thin filaments appeared, as demonstrated by immunogold for F-actin positivity. In cells exposed to hyperthermia, gold particles, suggestive of actin filaments, created a bridge between two diffuse chromatin areas, widely separated by the condensed chromatin (Figure $3 \mathrm{H}, \mathrm{I}$ ). No labeling has been found in the nucleoli, in condensed chromatin or in cup-shaped masses (Figure 3L). This localization suggests actin involvement in remodeling nuclear matrix during apoptotic cell death, without completely abandoning its principle localization site. In fact, in cells exposed to hyperthermia, gold particles also appeared in cytoplasm (Figure 3L).

\section{Discussion}

This work describes the HL-60 apoptotic cell features by a three-dimensional ultrastructural analysis with particular regard to nuclear events. Apoptosis induced by hyperthermia in HL-60 cells has been investigated by FEISEM, a scanning microscope at high definition and resolution. Interestingly, this innovative approach allowed to describe the three-dimensional chromatin behavior, its remodeling and cytoskeleton reorganization under apoptotic experimental condition.

Apoptotic chromatin condensation is not an active process as occurred in mitosis, but a consequence of a spatial rearrangement, which involved various cellular structures. As demonstrated by different authors ${ }^{30-32}$ chromatin during mitosis and interphase showed, by means of the FEISEM, a minor condensation level if compared to that observed in cup shaped masses and micronuclei. Moreover, to support this concept there is the fact that apoptotic chromatin, respect to the interphasic one, is cleaved and inactive. This finding evidences the gradualness of the apoptotic phenomenon that is opposed to the rapidity of the necrotic mechanisms. In fact in early apoptotic events it is possible to recognize chromatin areas which maintained an aspect and a role comparable to those of control cells. Moreover, this study show thin cytoskeleton filament involvement in early apoptotic stages as described by several author, but no data are present in literature regarding their three-dimensional organization. ${ }^{33-35}$ Thus, actin could play an important role in regulating spatial chromatin behavior during apoptosis. It is known that actin polymerizes actively inside the nucleus after apoptotic stimuli, partially leaving the cytoplasm where it exists in depolymerized form. Actin filaments, recognized by the immunogold reaction, are detected in diffuse chromatin areas where they formed a bridge above the condensed chromatin. Gold particles appear exclusively localized in diffuse chromatin and are strictly absent in condensed chromatin, cup-shaped masses and nucleoli. In hyperthermia-treated cells the diffuse chromatin areas with an aspect similar to those of the control cells, seem to represent the actin action site. In particular, actin serves as a point of contact between two zones of diffuse chromatin separated by condensed chromatin area. This result strongly suggests a role of nuclear actin filaments to achieve the wellknown spatial separation between the normalfunctional chromatin and that inactivated by the apoptotic process. Diffuse chromatin can be considered, in fact, a site where the nuclear matrix components are well recognized, and our data strongly suggest a dispersed chromatin organization comparable with that present in normal nucleus, where it is still possible evaluate a proper relationship with the nuclear matrix. Thus, also under apoptotic condition, actin seems to be a crucial protein which interacts with nuclear components, particularly with matrix proteins and diffuse chromatin. ${ }^{34}$ Some authors support the hypothesis that nuclear actin involvement in chromatin remodeling is associated with transcriptional processes during active cell death. . $^{34,36,37}$

These findings further confirm the relationship between nuclear matrix, actin, and diffuse chromatin, in which these structures actively cooperate. Knowledge of chromatin structures and the morpho-functional analysis of its fibrillar network has allowed to deter-
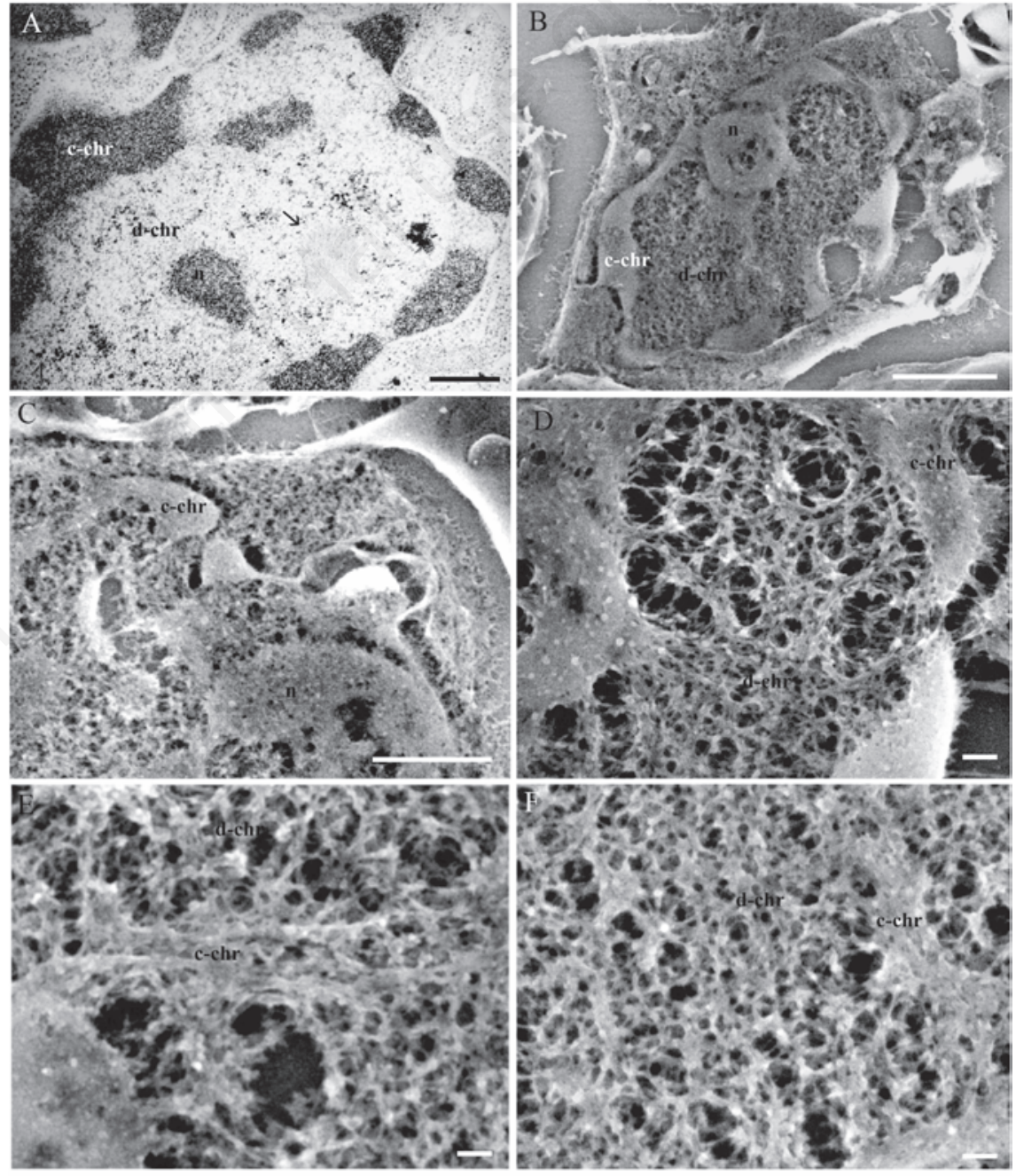

Figure 2. HL-60 cells exposed to hyperthermia observed at TEM (A) and FEISEM in SE image (B-F). Condensed chromatin organized in cup-shaped masses appears $(A, B)$ well separated but strictly connected $(C, D)$ with the diffuse chromatin $(E)$, that is similar to the control samples (F). n, nucleolus; c-chr, condensed chromatin; d-chr, diffuse chromatin; arrow, actin filaments. Scale bars: A) $0.5 \mu \mathrm{m}$; B,C) $1 \mu \mathrm{m}$; D-F) $100 \mathrm{~nm}$. 

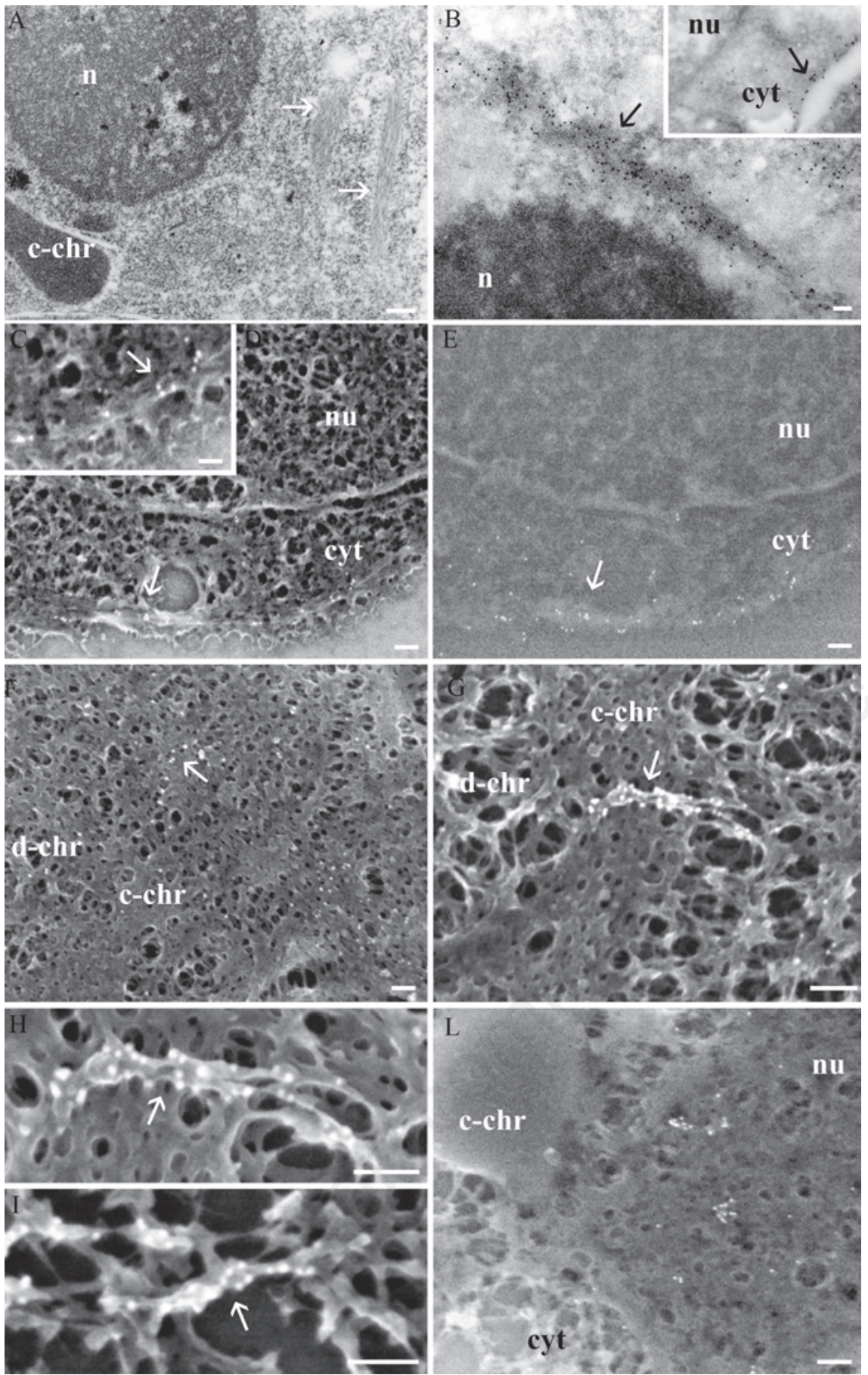

Figure 3. F-actin localization in HL-60 cells exposed to hyperthermia, at TEM $(A, B)$ and FEISEM (C-I). Filament bundles, suggestive of actin protein, appear in apoptotic HL60 cells, and have been then localized by means of immunogold reaction in the diffuse chromatin (B). Immunostaining can be observed in cytoplasm, and absent inside nucleus in control condition (inset in B). At FEISEM, in control cells F-actin localized in the cytoplasm along the plasma membrane where the gold particle can be detected (C-E, C,D: SE and BE image mix; D, pure BE image). After hyperthermia, apoptotic cells show actin filament presence inside nucleus, in the diffuse chromatin $(F)$ where gold particles appear organized to form a bridge between two diffuse chromatin areas separated by a condensed one (G-I; all $\mathrm{SE}$ and $\mathrm{BE}$ mix). Condensed chromatin appears negative to immunogold staining $(L) . n$, nucleolus; nu, nucleus; c-chr, condensed chromatin; $\mathrm{d}$-chr, diffuse chromatin; arrows, actin filaments; cty, cytoplasm. Scale bars: A,B,D-L) $100 \mathrm{~nm}$; C) $200 \mathrm{~nm}$. mine nuclear matrix role in the nucleus in interphase and during mitosis, potentially comparable to that played by actin in the apoptotic cells. Indeed, if nuclear matrix has the main role in maintaining nuclear architecture, actin protein associated with the nucleoskeleton could modulate and drive nuclear matrix in the spatial distribution of different chromatin forms in early apoptotic nucleus (chromatin condensation, cup-shaped masses and micronuclei formation), before later events (blebbing, apoptoic bodies and cell fragmentation without membrane integrity lost) that, irreversibly lead to programmed cell death.

Given the important role of actin cytoskeleton in cellular homeostasis, it is not surprising that it also has an important role in apoptosis. In fact, some authors demonstrate actin active role in initiating and mediating mammalian apoptosis via the intrinsic and extrinsic pathways and final degradation of actin filaments amplifies the apoptosis signaling cascade. ${ }^{38,39}$ Actin cytoskeleton has been demonstrated essential player during multiple hallmarks of apoptosis with dramatic changes in actin filament organization accompanying different stages of apoptosis. ${ }^{40,41}$ The important role of actin in the morphological hallmarks of apoptosis is coupled with mounting evidence demonstrating actin as a mediator and initiator of apoptosis signaling. ${ }^{42}$

Finally, this study highlights the apoptotic chromatin three-dimensional architecture and actin behavior during hyperthermia in HL-60 cells. Thus, to know actin rearrangements and its precise localization and spatial organization during early apoptotic events appear an important result and make thin cytoskeletal filaments a promising target for therapeutic intervention. ${ }^{26}$

\section{References}

1. Khan N, Adhami VM, Mukhtar H. Apoptosis by dietary agents for prevention and treatment of cancer. Biochem Pharmacol 2008;76:1333-9.

2. Chen F, Wang W, El-Deiry WS. Current strategies to target p53 in cancer. Biochem Pharmacol 2010;80:724-30.

3. Hildebrandt B, Wust P, Ahlers 0, Dieing A, Sreenivasa G, Kerner T, et al. The cellular and molecular basis of hyperthermia. Crit Rev Oncol Hematol 2002;43:33-56.

4. Falcieri E, Luchetti F, Burattini S, Canonico B, Santi S, Papa S. Lineagerelated sensitivity to apoptosis in human tumor cells undergoing hyperthermia. Histochem Cell Biol 2000;113:135-44.

5. Luchetti F, Burattini S, Ferri P, Papa S, Falcieri E. Actin involvement in apoptotic 
chromatin changes of hemopoietic cells undergoing hyperthermia. Apoptosis 2002;7:143-52.

6. Battistelli M, Salucci S, Olivotto E, Facchini A, Minguzzi M, Guidotti S, et al. Cell death in human articular chondrocyte: a morpho-functional study in micromass model. Apoptosis 2014;19:1471-83.

7. Battistelli M, Salucci S, Guescini M, Curzi D, Stocchi V, Falcieri E. Skeletal muscle cell behavior after physical agent treatments. Curr Pharm Des 2015 [Epub ahead of print].

8. Ahn YT, Shin IJ, Kim JM, Kim YS, Lee C, Ju SA, et al. Counteracting the activation of pAkt by inhibition of MEK/Erk inhibition reduces actin disruption-mediated apoptosis in PTEN-null PC3M prostate cancer cell lines. Oncol Lett 2013,6:1383-9.

9. Qi T, Tang W, Wang L, Zhai L, Guo L, Zeng $\mathrm{X}$. G-actin participates in RNA polymerase II-dependent transcription elongation by recruiting positive transcription elongation factor b (P-TEFb). J Biol Chem 2011;286:15171-81.

10. Pollard TD, Blanchoin L, Mullins RD. Molecular mechanisms controlling actin filament dynamics in nonmuscle cells. Annu Rev Biophys Biomol Struct 2000;29: 545-76.

11. Amann KJ, Pollard TD. Cellular regulation of actin network assembly. Curr Biol 2000;10:728-30.

12. dos Remedios CG, Chhabra D, Kekic M, Dedova IV, Tsubakihara M, Berry DA, et al. Actin binding proteins: regulation of cytoskeletal microfilaments. Physiol Rev 2003;83:433-73.

13. Paavilainen VO, Bertling E, Falck S, Lappalainen P. Regulation of cytoskeletal dynamics by actin-monomer-binding proteins, Trends Cell Biol 2004;14:386-394.

14. Ascough KR, Endocytosis: actin in the driving seat. Curr Biol 2004;14:R124-R126.

15. Carlier MF, Pantaloni D. Control of actin assembly dynamics in cell motility. J Biol Chem 2007;282:23005-9.

16. Shumaker DK, Kuczmarski ER, Goldman $\mathrm{RD}$. The nucleoskeleton: lamins and actin are major players in essential nuclear functions. Curr Opin Cell Biol 2003;15:358-366.

17. Bettinger BT, Gilbert DM, Amberg DC. Actin up in the nucleus. Nat Rev Mol Cell Biol 2004;5:410-5.
18. Kapoor P, Shen X. Mechanisms of nuclear actin in chromatin-remodeling complexes. Trends Cell Biol 2014;24:238-46.

19. Miralles F, Visa N. Actin in transcription and transcription regulation, Curr Opin Cell Biol 2006;18:261-6.

20. Chen M, Shen X. Nuclear actin and actinrelated proteins in chromatin dynamics. Curr Opin Cell Biol 2007;19:326-30.

21. Ferrai C, Naum-Onganía G, Longobardi E, Palazzolo M, Disanza A, Diaz VM, et al. Induction of HoxB transcription by retinoic acid requires actin polymerization. Mol Biol Cell 2009;20:3543-3551.

22. Jockusch BM, Schoenenberger CA, Stetefeld J, Aebi U. Tracking down the different forms of nuclear actin. Trends Cell Biol 16;2006:391-6.

23. Ye J, Zhao J, Hoffmann-Rohrer U, Grummt I. Nuclear myosin I acts in concert with polymeric actin to drive RNA polymerase I transcription. Genes Dev 2008;22:322-30.

24. Visa N, Percipalle P. Nuclear functions of actin. Cold Spring Harb Perspect Biol 2010;2:a000620.

25. Grzanka A, Grzanka D, Orlikowska M. Cytoskeletal reorganization during process of apoptosis induced by cytostatic drugs in K-562 and HL-60 leukemia cell lines. Biochem Pharmacol 2003;66:1611-7.

26. Grzanka A, Grzanka D, Zuryń A, Grzanka AA, Safiejko-Mroczka B. Reorganization of actin in K-562 and HL-60 cells treated with taxol. Neoplasma 2006;53:56-61.

27. Grzanka D, Marszałek A, Izdebska M, Gackowska L, Andrzej Szczepanski M, Grzanka A. Actin cytoskeleton reorganization correlates with cofilin nuclear expression and ultrastructural changes in cho aa8 cell line after apoptosis and mitotic catastrophe induction by doxorubicin. Ultrastruct Pathol 2011;35:130-8.

28. Burattini S, Ferri P, Battistelli M, D'Emilio A, Biagiotti L, Sestili P, et al. Apoptotic DNA fragmentation can be revealed in situ: an ultrastructural approach. Microsc Res Tech 2009;72:913-23.

29. Salucci S, Baldassarri V, Falcieri E, Burattini S. $\alpha$-Actinin involvement in Zdisk assembly during skeletal muscle C2C12 cells in vitro differentiation. Micron 2015;68:47-53.

30. Gobbi P, Falconi M, Vitale M, Galanzi A, Artico M, Martelli AM, Mazzotti G. Scanning electron microscopic detection of nuclear structures involved in DNA replication. Arch Histol Cytol 1999;62:317-26.

31. Gobbi P, Thalhammer S, Falconi M, Stark R, Heckl WM, Mazzotti G. Correlative high resolution morphological analysis of the three dimensional organization of human metaphase chromosomes. Scanning 2000; 22:273-81.

32. Falconi M, Teti G, Zago M, Pelotti S, Gobbi P, Breschi L, et al. Effect of fixative on chromatin structure and DNA detection. Microsc Res Tech 2007;70:599-606.

33. Grzanka D, Gagat M, Izdebska M. Actin is required for cellular death. Acta Histochem 2013;115:775-82.

34. Grzanka D, Gagat M, Izdebska M. Involvement of the SATB1/F-actin complex in chromatin reorganization during active cell death. Int J Mol Med 2014;33:1441-50.

35. Izdebska M, Gagat M, Grzanka D, Grzanka A. Ultrastructural localization of F-actin using phalloidin and quantum dots in HL60 promyelocytic leukemia cell line after cell death induction by arsenic trioxide. Acta Histochem 2013;115:487-95.

36. Gotzmann J, Meissner M, Gerner C. The fate of the nuclear matrix-associatedregion-binding protein SATB1 during apoptosis. Cell Death Differ 2000;7:425-38.

37. Hofmann WA, Stojiljkovic L, Fuchsova B, Vargas GM, Mavrommatis E, Philimonenko $\mathrm{V}$, et al. Actin is part of pre-initiation complexes and is necessary for transcription by RNA polymerase II. Nat Cell Biol 2004; 6:1094-101.

38. Elmore S. Apoptosis: a review of programmed cell death. Toxicol Pathol 2007; 35:495-516.

39. Sekino Y, Kojima N, Shirao T. Role of actin cytoskeleton in dendritic spine morphogenesis. Neurochem Int 2007;51:92-104.

40. Häcker G. The morphology of apoptosis. Cell Tissue Res 2000;301:5-17.

41. Laster SM, Mackenzie JM Jr. Bleb formation and F-actin distribution during mitosis and tumor necrosis factor-induced apoptosis. Microsc Res Tech 1996;34:272-80.

42. Desouza M, Gunning PW, Stehn JR. The actin cytoskeleton as a sensor and mediator of apoptosis. Bioarchitecture 2012;2: 75-87. 